TRANSACTIONS OF THE

AMERICAN MATHEMATICAL SOCIETY

Volume 356, Number 11, Pages 4643-4653

S 0002-9947(04)03506-8

Article electronically published on January 23, 2004

\title{
VISCOSITY SOLUTIONS, ALMOST EVERYWHERE SOLUTIONS AND EXPLICIT FORMULAS
}

\author{
BERNARD DACOROGNA AND PAOLO MARCELLINI
}

\begin{abstract}
Consider the differential inclusion $D u \in E$ in $\mathbb{R}^{n}$. We exhibit an explicit solution that we call fundamental. It also turns out to be a viscosity solution when properly defining this notion. Finally, we consider a Dirichlet problem associated to the differential inclusion and we give an iterative procedure for finding a solution.
\end{abstract}

\section{INTRODUCTION}

Existence of almost everywhere solutions of the first order Dirichlet problem related to implicit differential equations of the type

$$
\left\{\begin{array}{cc}
F(D u(x))=0, & \text { a.e. } x \in \Omega, \\
u(x)=0, & x \in \partial \Omega,
\end{array}\right.
$$

has recently been extensively studied in the book [6] by the authors. Here $F$ : $\mathbb{R}^{n} \rightarrow \mathbb{R}$ is a continuous function and we look for a Lipschitz-continuous solution $u: \Omega \subset \mathbb{R}^{n} \rightarrow \mathbb{R}$. A wide literature on this subject can be found in [6], not only for scalar problems such as this one, but also for vector-valued solutions of first order systems related to maps $u: \Omega \subset \mathbb{R}^{n} \rightarrow \mathbb{R}^{m}$ and $F: \mathbb{R}^{m \times n} \rightarrow \mathbb{R}^{N}$, for some $m, N \geq 1$.

Existence of viscosity solutions of the Dirichlet problem (11) is now well established. It has been studied by many authors starting with Hopf, Lax, Kruzkov and Crandall-Lions; see for example [1] or [6] for more historical comments. One of the earliest and still one of the most complete monographs on the subject is [10 by P.L. Lions. The research in this field remains very active; in particular H. Ischii and P. Loreti [8], motivated by an optimization problem, recently gave an existence result of viscosity solutions of the Dirichlet problem (11). See also [2] and [9.

In this paper we give some existence results, either in the case of almost everywhere solutions, or, when possible, of viscosity solutions. One of our aims is to give some constructive explicit formulas (cf. Theorems 1 and 6 ). Moreover, if the geometry of the set $\Omega$ and the assumptions on the function $F$ make it possible, following [3] we give (cf. Corollary [8) an explicit formula for a viscosity solution of the Dirichlet problem (1), simply in terms of sup and inf. Otherwise, with general $F$ and $\Omega$, we propose, in Section 4 , an iteration scheme for characterizing a solution.

Received by the editors December 2, 2002 and, in revised form, August 21, 2003.

2000 Mathematics Subject Classification. Primary 34A60, 35F30, 49L25.

Key words and phrases. Almost everywhere solutions, viscosity solutions of nonlinear first order partial differential equations. 
In Section 2 we introduce the notion of viscosity solution of a differential inclusion; namely: given a closed set $E$, we say that a function $u$ is a viscosity solution of the differential inclusion

$$
D u(x) \in E, x \in \Omega,
$$

if $u$ is a viscosity solution of the equation

$$
F(D u(x))=0, x \in \Omega,
$$

where $F(\xi)=\operatorname{dist}\{\xi, E\}$. We will prove in Theorem 6 that the function $L: \mathbb{R}^{n} \rightarrow$ $\mathbb{R}$, defined by

$$
L(x)=\max \{\langle\xi, x\rangle: \xi \in E\},
$$

is a viscosity solution of the differential inclusion (2), i.e. it is a fundamental solution of the equation (3).

\section{Fundamental solution And Viscosity solutions OF DIFFERENTIAL INCLUSIONS}

We start by recalling some classical definitions and notations in convex analysis. We say that $\xi \in \mathbb{R}^{n}$ is an extreme point for a convex set $K \subset \mathbb{R}^{n}$ if the conditions

$$
\left\{\begin{array}{l}
\xi=t \xi_{1}+(1-t) \xi_{2} \\
\xi_{1}, \xi_{2} \in K, \quad t \in(0,1)
\end{array}\right.
$$

imply that $\xi=\xi_{1}=\xi_{2}$.

If $E$ is a set (not necessarily convex) of $\mathbb{R}^{n}$, we denote by $E_{\text {ext }}$ the set of extreme points of the convex hull of $E$ denoted by co $E$ (note that $E_{\text {ext }} \subset E$ ).

We also recall that the domain of a convex function $L: \mathbb{R}^{n} \rightarrow \mathbb{R} \cup\{+\infty\}$ is defined as

$$
\operatorname{dom} L=\left\{x \in \mathbb{R}^{n}: L(x)<+\infty\right\} .
$$

Theorem 1 below generalizes an analogous result obtained by Ischii and Loreti (see the proof of Theorem 2.2 in [8]) in the case that $E$ is the level set of a continuous, positively homogeneous function of degree one, equal to zero only at the origin of $\mathbb{R}^{n}$.

Theorem 1. Let $E$ be a compact set of $\mathbb{R}^{n}$. For every $x \in \mathbb{R}^{n}$ let

$$
L(x)=\max \{\langle\xi, x\rangle: \xi \in E\} .
$$

Then

$$
D L(x) \in E \text { a.e. } x \in \mathbb{R}^{n} .
$$

Remark 2. (i) It should be noted that in fact the theorem is more precise, namely

$$
D L(x) \in \overline{E_{\text {ext }}} \subset E \cap \partial \operatorname{co} E \text { a.e. } x \in \mathbb{R}^{n} \text {. }
$$

(ii) If $E$ is any set, not necessarily closed or bounded, then the proof gives (replacing max by sup) that

$$
D L(x) \in \bar{E} \text { a.e. } x \in \operatorname{dom} L \text {. }
$$

(iii) In terms of convex analysis and anticipating on (4) we can say that $L$ is the support function of co $E$.

Before proceeding with the proof it might be interesting to rewrite the theorem in terms of equations. 
Corollary 3. Let $F: \mathbb{R}^{n} \rightarrow \mathbb{R}$ be a continuous function such that

$$
E=\left\{\xi \in \mathbb{R}^{n}: F(\xi)=0\right\}
$$

is a bounded set. Let $L(x)=\max \{\langle\xi, x\rangle: F(\xi)=0\}$; then

$$
F(D L(x))=0 \text { a.e. } x \in \mathbb{R}^{n} \text {. }
$$

Proof. The following representation formula for $L$ holds (see Rockafellar [12], Theorem 32.2$)$ :

$$
L(x)=\max \{\langle\xi, x\rangle: \xi \in \operatorname{co} E\}=\max \{\langle\xi, x\rangle: \xi \in E\}, \quad \forall x \in \mathbb{R}^{n} .
$$

In fact one has the more precise result (see Rockafellar [12], Corollary 32.3.2)

$$
L(x)=\max \{\langle\xi, x\rangle: \xi \in \operatorname{co} E\}=\max \left\{\langle\xi, x\rangle: \xi \in E_{\text {ext }}\right\}, \quad \forall x \in \mathbb{R}^{n} .
$$

Let $\left\{\xi_{h}\right\}_{h \in \mathbb{N}}$ be a (finite or) countable dense subset of $E_{\text {ext }} \subset E$ and, analogously to (4), for every $h \in \mathbb{N}$ and for every $x \in \mathbb{R}^{n}$ let us define

$$
L_{h}(x)=\max \left\{\left\langle\xi_{1}, x\right\rangle,\left\langle\xi_{2}, x\right\rangle, \ldots,\left\langle\xi_{h}, x\right\rangle\right\} .
$$

Clearly the gradient $D L_{h}$ exists almost everywhere in $\mathbb{R}^{n}$ and

$$
D L_{h}(x) \in\left\{\xi_{1}, \xi_{2}, \ldots, \xi_{h}\right\} \subset E_{\text {ext }}, \quad \text { a.e. } x \in \mathbb{R}^{n} .
$$

For every $x \in \mathbb{R}^{n}$ the sequence $L_{h}(x)$ is increasing with respect to $h \in \mathbb{N}$ and we have

$$
L(x)=\sup \left\{L_{h}(x): h \in \mathbb{N}\right\}=\lim _{h \rightarrow+\infty} L_{h}(x) .
$$

For every $h \in \mathbb{N}$ the sequence $L_{h}(x)$ is convex with respect to $x \in \mathbb{R}^{n}$ and

$$
\operatorname{dom} L_{h}=\operatorname{dom} L=\mathbb{R}^{n} .
$$

Thus we can apply Lemma 4 and we obtain that, at every point where $L_{h}$ and $L$ are differentiable (i.e., almost everywhere in $\mathbb{R}^{n}$ ),

$$
D L_{h}(x) \rightarrow D L(x) .
$$

Therefore, by (6), we get the conclusion

$$
D L(x) \in \overline{E_{\text {ext }}} \subset E, \quad \text { a.e. } x \in \mathbb{R}^{n} .
$$

In the proof of Theorem 1 we used a result given in 11 (Lemma 5.9), that we recall here in a form more appropriate to the applications given in this paper.

Lemma 4. Let $\left\{L_{h}\right\}_{h \in \mathbb{N}}$ be a sequence of convex functions, defined on $\mathbb{R}^{n}$ with values on $\mathbb{R} \cup\{+\infty\}$, with pointwise limit $L: \mathbb{R}^{n} \rightarrow \mathbb{R} \cup\{+\infty\}$. At every point $x \in \operatorname{int}\left[\left(\bigcap_{h \in \mathbb{N}} \operatorname{dom} L_{h}\right) \cap \operatorname{dom} L\right]$, where $L_{h}$ and $L$ are differentiable, the gradient $D L_{h}(x)$ converges in $\mathbb{R}^{n}$ to the gradient $D L(x)$.

Proof. For every $h \in \mathbb{N}$ let $\operatorname{dom} L_{h}$ and $\operatorname{dom} L$ be the domains of $L_{h}$ and $L$. Then each $L_{h}$ is locally Lipschitz-continuous in $\operatorname{int} \operatorname{dom} L_{h}$ and $L$ is locally Lipschitzcontinuous in int $\operatorname{dom} L$. Therefore, for every $h \in \mathbb{N}$, there exists a set $N_{h} \subset$ $\operatorname{dom} L_{h} \subset \mathbb{R}^{n}$ of zero measure such that $L_{h}$ is differentiable at every point of $\operatorname{dom} L_{h} \backslash N_{h}$. Analogously, there exists a set $N \subset \operatorname{dom} L$ of zero measure such that 
$L$ is differentiable at every point of $\operatorname{dom} L \backslash N$. Then the set of points $x \in \mathbb{R}^{n}$ where $L_{h}$ and $L$ are differentiable is (possibly empty and) given by

$$
\left(\bigcap_{h \in \mathbb{N}}\left(\operatorname{dom} L_{h} \backslash N_{h}\right)\right) \bigcap(\operatorname{dom} L \backslash N)
$$

and differs from the intersection of their domains $\left(\bigcap_{h \in \mathbb{N}} \operatorname{dom} L_{h}\right) \cap \operatorname{dom} L$ by (at most) a set $\left(\bigcup_{h \in \mathbb{N}} N_{h}\right) \cup N$ of zero measure. Let $x \in \operatorname{int}\left[\left(\bigcap_{h \in \mathbb{N}} \operatorname{dom} L_{h}\right) \cap \operatorname{dom} L\right]$ be a point of $\mathbb{R}^{n}$ where $L_{h}$ and $L$ are differentiable. Let $i \in\{1,2, \ldots, n\}$ and $h \in \mathbb{N}$ be fixed. Then at $x=\left(x_{1}, \ldots, x_{i}, \ldots, x_{n}\right)$ the partial derivatives $\partial L_{h} / \partial x_{i}$ and $\partial L / \partial x_{i}$ are well defined. An elementary application of the convex inequality for the function $L_{h}$ gives the monotonicity of the difference quotient; precisely, if $t>0$ is sufficiently small and if, as usual, we denote by $x \pm t e_{i}$ the two points of $\mathbb{R}^{n}$ with coordinates respectively $\left(x_{1}, \ldots, x_{i-1}, x_{i} \pm t, x_{i+1}, \ldots, x_{n}\right)$, we have

$$
\frac{L_{h}\left(x-t e_{i}\right)-L_{h}(x)}{-t} \leq \frac{\partial L_{h}}{\partial x_{i}}(x) \leq \frac{L_{h}\left(x+t e_{i}\right)-L_{h}(x)}{t}
$$

and, in the limit as $h \rightarrow+\infty$,

$$
\frac{L\left(x-t e_{i}\right)-L(x)}{-t} \leq \liminf _{h \rightarrow+\infty} \frac{\partial L_{h}}{\partial x_{i}}(x) \leq \limsup _{h \rightarrow+\infty} \frac{\partial L_{h}}{\partial x_{i}}(x) \leq \frac{L\left(x+t e_{i}\right)-L(x)}{t} .
$$

Since $L$ is differentiable at $x$, as $t \rightarrow 0^{+}$we obtain that $\partial L_{h} / \partial x_{i}(x)$ converges to $\partial L / \partial x_{i}$. The property being such that for every $i \in\{1,2, \ldots, n\}$, we have the conclusion, i.e., that the gradient $D L_{h}(x)$ converges in $\mathbb{R}^{n}$ to the gradient $D L(x)$.

Remark 5. With a slightly different proof, as in Lemma 5.9 in [11, we can give a compactness result. Precisely, we can show that from every locally bounded sequence $\left\{L_{h}\right\}_{h \in \mathbb{N}}$ of convex functions $\left(\left\{L_{h}\right\}_{h \in \mathbb{N}}\right.$ uniformly bounded in $L_{\text {loc }}^{\infty}(\Omega)$, with $\Omega$ open set in $\left.\mathbb{R}^{n}\right)$ it is possible to select a subsequence $\left\{L_{h_{k}}\right\}_{k \in \mathbb{N}}$ whose gradients $\left\{D L_{h_{k}}\right\}_{k \in \mathbb{N}}$ converge almost everywhere in $\Omega$, and at the same time $\left\{L_{h_{k}}\right\}_{k \in \mathbb{N}}$ converges in the strong topology of $W_{\text {loc }}^{1, q}(\Omega)$, for every $q \in[1,+\infty)$.

With the help of the above construction we can give a definition of what we mean by viscosity solutions of differential inclusions. Given a closed set $E$, we say that a function $u$ is a viscosity solution of the differential inclusion

$$
D u(x) \in E, x \in \mathbb{R}^{n},
$$

if $u$ is a viscosity solution of the equation

$$
F(D u(x))=0, x \in \mathbb{R}^{n},
$$

where $F(\xi)=\operatorname{dist}\{\xi, E\}$. We therefore have the following result.

Theorem 6. Let $E$ be a compact set of $\mathbb{R}^{n}$. For every $x \in \mathbb{R}^{n}$ let

$$
L(x)=\max \{\langle\xi, x\rangle: \xi \in E\} .
$$

Then $L$ is a viscosity solution of

$$
D L(x) \in E, x \in \mathbb{R}^{n} .
$$


Proof. The function $L$ being convex we have that $D^{+} L(x)$ (the superdifferential of $L$ at $x$; see [1] and [6] for the precise definition of this set) is either empty or reduced to $\{D L(x)\}$, i.e. $x$ is a point of differentiability of $L$ and we know by Theorem 1 that at such points $D L(x) \in E$. We therefore have that

$$
F(p)=0, \forall p \in D^{+} L(x),
$$

which means that $L$ is a viscosity subsolution (see Proposition 4.7 of [6]) of $F(D u)=$ 0 .

Since $F \geq 0$ we deduce trivially that

$$
F(p) \geq 0, \forall p \in D^{-} L(x),
$$

where $D^{-} L(x)$ is the subdifferential of $L$ at $x$. This means that $L$ is a viscosity supersolution of $F(D u)=0$.

Combining these two results we have indeed that $L$ is a viscosity solution of $F(D u)=0$ and hence of $D u \in E$.

\section{Fundamental SOlution AND the Boundary CONDition}

We now want to discuss a Dirichlet problem in a bounded domain. We first fix the notations.

We let $\Omega \subset \mathbb{R}^{n}$ be a bounded open convex set and denote by $\nu(y)$ the outward unit normal at $y \in \partial \Omega$ (that exists at almost all points $y \in \partial \Omega$, since $\Omega$ is convex).

We next let $E \subset \mathbb{R}^{n}$ be a compact set with $0 \in \operatorname{intco} E$. We then associate to co $E$ its gauge $\rho$, which is a convex and positively homogeneous of degree one function, such that

$$
\operatorname{co} E=\left\{\xi \in \mathbb{R}^{n}: \rho(\xi) \leq 1\right\} .
$$

Recall also that

$$
L(x)=\max \{\langle\xi, x\rangle: \xi \in E\} .
$$

We should immediately note that, with our hypotheses on $E$ (and invoking (4)), the function $L$ is in fact the polar of $\rho$, denoted also sometimes by $\rho^{0}$.

We finally consider the Dirichlet problem

$$
\left\{\begin{array}{cc}
D u(x) \in E, & \text { a.e. } x \in \Omega, \\
u(x)=0, & x \in \partial \Omega .
\end{array}\right.
$$

We could also consider the case of a more general boundary datum of class $C^{1}$ but the analysis can then be carried in a straightforward manner.

We have the following theorem that is inspired by Cardaliaguet-DacorognaGangbo-Georgy [3] (see also [6]).

Theorem 7. Let $\Omega, \nu, E, \rho$ and $L$ be as above and satisfy in addition

$$
\frac{-\nu(y)}{\rho(-\nu(y))} \in E, \text { a.e. } y \in \partial \Omega ;
$$

then the function $u: \mathbb{R}^{n} \rightarrow \mathbb{R}$, defined by

$$
u(x)=\min \{L(x-y): y \in \partial \Omega\},
$$

solves the Dirichlet problem

$$
\left\{\begin{array}{c}
D u(x) \in E, \quad \text { a.e. } x \in \Omega, \\
u(x)=0, \quad x \in \partial \Omega .
\end{array}\right.
$$

As before we rewrite this theorem in terms of functions. 
Corollary 8. Let $F: \mathbb{R}^{n} \rightarrow \mathbb{R}$ be continuous with $F(\xi) \rightarrow \infty$ as $|\xi| \rightarrow \infty$ and $F(0)<0$. Set

$$
E=\left\{\xi \in \mathbb{R}^{n}: F(\xi)=0\right\} .
$$

Let $\Omega, \nu, \rho$ and $L$ be as above. If

$$
F\left(\frac{-\nu(y)}{\rho(-\nu(y))}\right)=0 \text { a.e. } y \in \partial \Omega
$$

then

$$
u(x)=\min \{L(x-y): y \in \partial \Omega\}
$$

solves

$$
\left\{\begin{array}{cc}
F(D u(x))=0, & \text { a.e. } x \in \Omega \\
u(x)=0, & x \in \partial \Omega
\end{array}\right.
$$

Furthermore if $E \subset \partial \operatorname{co} E$, then $u$ is a viscosity solution.

Remark 9. (i) The first part of the corollary follows immediately from the theorem. The fact that $u$ is a viscosity solution (when $E \subset \partial \operatorname{co} E$ ) was established in [3].

(ii) Note that if, in addition, $\partial \operatorname{co} E \subset E$ (which happens if, for instance, $F$ is convex or more generally if the set $\{\xi: F(\xi) \leq 0\}$ is convex), then (10) is always satisfied. In fact, since $\rho$ is positively homogeneous of degree one,

$$
\rho\left(\frac{-\nu(y)}{\rho(-\nu(y))}\right)=1 \Rightarrow \frac{-\nu(y)}{\rho(-\nu(y))} \in \partial \operatorname{co} E \subset E .
$$

Moreover, if $E=\partial \operatorname{co} E, u$ defined in (11) is the unique viscosity solution of (12).

(iii) According to Theorem 4.1 of Lions [10], the Dirichlet problem (12) always has a viscosity solution. However the solution given by (11) is not necessarily a viscosity solution; it is so when $E \subset \partial \operatorname{co} E$.

We can now proceed with the proof of the theorem.

Proof of Theorem 7 . We recall the following two facts (the first one is just the HopfLax formula and the second one is Lemma 2.9 in [3] or Lemma 4.17 in [6]). We also use the standard notation $D^{+} u(x)$, respectively $D^{-} u(x)$, for the superdifferential, respectively the subdifferential, of $u$ at $x$ (see [6] for more details).

Fact 1: The function $u$ is the viscosity solution of

$$
\left\{\begin{array}{cc}
\rho(D u(x))=1, & x \in \Omega, \\
u(x)=0, & x \in \partial \Omega .
\end{array}\right.
$$

Fact 2: Let $y(x) \in \partial \Omega$ be such that

$$
u(x)=L(x-y(x)) .
$$

Then, if $p \in D^{-} u(x)$ (i.e. $D^{-} u(x)$ is non empty), the outward unit normal $\nu(y(x))$ is well defined and there exists $\lambda(y(x))>0$ such that

$$
p=-\lambda(y(x)) \nu(y(x)) .
$$

Since we are interested in almost everywhere solutions we need only to consider points $x \in \Omega$ where $D^{+} u(x)=D^{-} u(x)=\{D u(x)\}$. Combining (13) and (14) with $p=D u(x)$ and the homogeneity of $\rho$, we get that $\lambda(y)=1 / \rho(-\nu(y))$ and hence

$$
D u(x)=\frac{-\nu(y)}{\rho(-\nu(y))} .
$$

The hypothesis (7) leads to the result $D u \in E$. 


\section{The ITERATION SCHEME}

As above we let $\Omega \subset \mathbb{R}^{n}$ be a nonempty bounded open set. We want to find, with the help of the previous construction, a solution $u \in W_{0}^{1, \infty}(\Omega)$ of the differential inclusion

$$
D u(x) \in E \text {, a.e. } x \in \Omega,
$$

where $E \subset \mathbb{R}^{n}$ is a compact set with $0 \in \operatorname{intco} E$. We let $\rho$ be the gauge associated to co $E$.

We will find a sequence of disjoint convex open sets $\Omega_{i} \subset \Omega$ so that

$$
\operatorname{meas}\left[\Omega \backslash \bigcup_{i=1}^{\infty} \Omega_{i}\right]=0
$$

and the function $u$ will be defined as

$$
u(x)=\left\{\begin{array}{cl}
\inf \left\{L(x-y): y \in \partial \Omega_{i}\right\}, & x \in \Omega_{i}, \\
0, & x \in \Omega \backslash \bigcup_{i=1}^{\infty} \Omega_{i} .
\end{array}\right.
$$

Observe that $u$ is a viscosity solution of the Dirichlet problem $D u \in E$ in $\Omega_{i}, u=0$, on $\partial \Omega_{i}$ for every $i$ (but not globally in $\Omega$ ).

Any Vitali covering by level sets of the function $L$ has all the above requirements. However we will choose, among them, one with some maximality properties. In particular we want that $\Omega_{1}=\Omega$ if $\Omega$ is convex and $\frac{-\nu}{\rho(-\nu)} \in E$, a.e. on $\partial \Omega$, where $\nu$ is the outward unit normal to $\Omega$ (recall that this always happens if $E=\partial \operatorname{co} E$ or if $\Omega$ is the level set of the function $L)$.

Before describing this construction we need to introduce some notations.

Notation 10. Let $x_{0} \in \mathbb{R}^{n}$. We let $G_{x_{0}}$ be the set of all gauges centered at $x_{0}$. In other words this is the set of all convex functions $\gamma: \mathbb{R}^{n} \rightarrow \mathbb{R}$ satisfying

$$
\begin{gathered}
\gamma\left(x_{0}\right)=0, \gamma(x)>0, \forall x \in \mathbb{R}^{n} \backslash\left\{x_{0}\right\}, \\
\gamma\left(t\left(x-x_{0}\right)+x_{0}\right)=t \gamma(x), \forall x \in \mathbb{R}^{n}, \forall t>0 .
\end{gathered}
$$

Proposition 11. (i) If $\gamma \in G_{x_{0}}$ is differentiable at $x \in \mathbb{R}^{n}$ (this happens at almost all points), then it is differentiable at any $x_{t} \in \mathbb{R}^{n}$ of the form $x_{t}=t\left(x-x_{0}\right)+x_{0}$, $t>0$ and

$$
D \gamma\left(x_{t}\right)=D \gamma(x)
$$

In particular $\gamma$ is differentiable at almost all points of $\left\{x \in \mathbb{R}^{n}: \gamma(x)=1\right\}$.

(ii) Let $C \subset \mathbb{R}^{n}$ be a nonempty bounded open convex set and $x_{0} \in \operatorname{int} C$. The gauge of $C$ centered at $x_{0}$ is defined as

$$
\gamma_{C, x_{0}}(x)=\inf \left\{\lambda \geq 0: x_{0}+\frac{x-x_{0}}{\lambda} \in C\right\} .
$$

Then $\gamma_{C, x_{0}} \in G_{x_{0}}$ and

$$
\begin{aligned}
C & =\left\{x \in \mathbb{R}^{n}: \gamma_{C, x_{0}}(x)<1\right\}, \\
\partial C & =\left\{x \in \mathbb{R}^{n}: \gamma_{C, x_{0}}(x)=1\right\} .
\end{aligned}
$$

Remark 12. At almost every point $x \in \partial C, \gamma_{C, x_{0}}$ is differentiable and $D \gamma_{C, x_{0}}(x)$ is then an outward normal to $C$. 
We will proceed inductively to define $\Omega_{i}$. We start by choosing a sequence of points in $\Omega,\left\{x^{N}\right\}_{N=1}^{\infty}$, dense in $\Omega$. We set $\Omega_{0}=\emptyset$ and assume that $\Omega_{i}$ has already been defined. If $\Omega \backslash \bigcup_{k=0}^{i} \bar{\Omega}_{k}=\emptyset$, then the procedure is already over. We then define, $N=N(i+1)$,

$$
N(i+1)=\min \left\{N: x^{N} \in \Omega \backslash \bigcup_{k=0}^{i} \bar{\Omega}_{k}\right\}
$$

and we label $x_{i+1}=x^{N(i+1)}$ (so that $\left.x_{1}=x^{1}\right)$. We then choose $r_{i+1}>0$ sufficiently small so that

$$
\left\{x \in \mathbb{R}^{n}: l_{r_{i+1}}(x) \equiv \frac{L\left(x_{i+1}-x\right)}{r_{i+1}}<1\right\} \subset \Omega \backslash \bigcup_{k=0}^{i} \bar{\Omega}_{k},
$$

where

$$
L(x)=\max \{\langle\xi, x\rangle: \xi \in E\} .
$$

This is always possible since $\Omega \backslash \bigcup_{k=0}^{i} \bar{\Omega}_{k}$ is an open set, $x_{i+1} \in \Omega \backslash \bigcup_{k=0}^{i} \bar{\Omega}_{k}, L(0)=0$ and $L$ is locally Lipschitz.

We next define

$$
\begin{aligned}
& \Gamma\left(x_{i+1}, \Omega \backslash \bigcup_{k=0}^{i} \bar{\Omega}_{k}\right) \\
& =\left\{\begin{array}{c}
\gamma \in G_{x_{i+1}}: \frac{-D \gamma(x)}{\rho(-D \gamma(x))} \in E, \text { a.e. } x \in \mathbb{R}^{n} \\
\left\{x \in \mathbb{R}^{n}: l_{r_{i+1}}(x)<1\right\} \subset\left\{x \in \mathbb{R}^{n}: \gamma(x)<1\right\} \subset \Omega \backslash \bigcup_{k=0}^{i} \bar{\Omega}_{k}
\end{array}\right\} .
\end{aligned}
$$

Note that $l_{r_{i+1}} \in \Gamma\left(x_{i+1}, \Omega \backslash \bigcup_{k=0}^{i} \bar{\Omega}_{k}\right)$, since, by Theorem廿 $D L \in E$ and $\rho(D L)=$ 1. Observe also that if $\gamma \in \Gamma\left(x_{i+1}, \Omega \backslash \bigcup_{k=0}^{i} \bar{\Omega}_{k}\right)$, then

$$
\gamma \leq l_{r_{i+1}} .
$$

We now claim that there exists $\gamma_{i+1} \in \Gamma\left(x_{i+1}, \Omega \backslash \bigcup_{k=0}^{i} \bar{\Omega}_{k}\right)$ such that if

$$
\Omega_{i+1}=\left\{x \in \mathbb{R}^{n}: \gamma_{i+1}(x)<1\right\}
$$

then

$$
\operatorname{meas}\left(\Omega_{i+1}\right)=\sup _{\gamma \in \Gamma\left(x_{i+1}, \Omega \backslash \bigcup_{k=0}^{i} \bar{\Omega}_{k}\right)}\left[\operatorname{meas}\left\{x \in \mathbb{R}^{n}: \gamma(x)<1\right\}\right] .
$$

Indeed let $\left\{\gamma^{s}\right\}$ be a maximizing sequence. From (15), we deduce that up to a subsequence, that we still label $\left\{\gamma^{s}\right\}$, the sequence converges to an element $\gamma_{i+1} \in$ $\Gamma\left(x_{i+1}, \Omega \backslash \bigcup_{k=0}^{i} \bar{\Omega}_{k}\right)$. In fact all the conditions are easily checked. By Remark 5 we have

$$
\frac{-D \gamma_{i+1}(x)}{\rho\left(-D \gamma_{i+1}(x)\right)} \in E
$$


Let us prove, for example, that $\gamma_{i+1}(x) \neq 0$ if $x \neq x_{i+1}$. Assume for the sake of contradiction that there exists $y \neq x_{i+1}$ with $\gamma_{i+1}(y)=0$. We would deduce that $\gamma_{i+1} \equiv 0$ on the half line $x_{i+1}+t\left(y-x_{i+1}\right), t \geq 0$, which contradicts, $\Omega$ being bounded, the inclusion $\left\{x \in \mathbb{R}^{n}: \gamma_{i+1}(x)<1\right\} \subset \Omega$.

Since the measure is upper semicontinuous (in fact even continuous), cf. Proposition 14 with respect to the type of convergence under consideration we have the result.

Observe that, as wished, $\Omega_{1}=\Omega$ if $\Omega$ is convex and $\frac{-\nu}{\rho(-\nu)} \in E$, a.e. on $\partial \Omega$ (because choosing $\omega$ the gauge of $\Omega$ centered at $x_{1}$, we would have $\omega \in \Gamma\left(x_{1}, \Omega\right)$ ).

Since we have, with this procedure, exhausted all elements of the sequence $\left\{x^{N}\right\}$, we have indeed

$$
\operatorname{meas}\left[\Omega \backslash \bigcup_{i=1}^{\infty} \bar{\Omega}_{i}\right]=0 \text {. }
$$

Example 13. Consider the case $\Omega=(-1,1)^{2} \subset \mathbb{R}^{2}, u=u\left(x_{1}, x_{2}\right)$ and

$$
\left\{\begin{array}{cc}
\left(\left(\frac{\partial u}{\partial x_{1}}\right)^{2}-1\right)^{2}+\left(\left(\frac{\partial u}{\partial x_{2}}\right)^{2}-1\right)^{2}=0 & \text { a.e. in } \Omega \\
u=0 & \text { on } \partial \Omega .
\end{array}\right.
$$

Choosing the grid sequence $\left\{x^{N}\right\}_{N=1}^{\infty}$ in a suitable way, starting with $x^{1}=(0,0)$, we find with our procedure

$$
\Omega^{1}=\left\{x \in \mathbb{R}^{2}:\left|x_{1}\right|+\left|x_{2}\right| \leq 1\right\} \text { and } u\left(x_{1}, x_{2}\right)=1-\left|x_{1}\right|-\left|x_{2}\right| \text { in } \Omega_{1} .
$$

Similarly for $\Omega_{i}$. Our construction is compatible with the numerical computations of [4].

We end up with an elementary convergence result that we used above.

Proposition 14. Let $\left\{\gamma^{s}\right\}_{s \in \mathbb{N}}$ and $\gamma^{\infty}$ be measurable functions defined on a bounded measurable set $\Omega \subset \mathbb{R}^{n}$. Let

$$
\begin{aligned}
\Omega^{s} & =\left\{x \in \Omega: \gamma^{s}(x) \leq 1\right\}, \\
\Omega^{\infty} & =\left\{x \in \Omega: \gamma^{\infty}(x) \leq 1\right\} .
\end{aligned}
$$

If $\gamma^{s} \rightarrow \gamma^{\infty}$ a.e. in $\Omega$, then

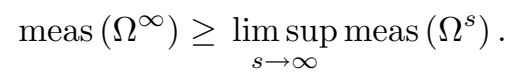

If, in addition, $\gamma^{s}$ and $\gamma^{\infty}$ are gauges centered at $x_{0} \in \Omega$ and $\Omega$ is open, then

$$
\text { meas }\left(\Omega^{\infty}\right)=\lim _{s \rightarrow \infty} \operatorname{meas}\left(\Omega^{s}\right)
$$

Remark 15. Note that if $\gamma^{s}$ and $\gamma^{\infty}$ are merely convex, then continuity does not hold, as the following example shows. Let $\Omega \subset \mathbb{R}^{n}$ be a bounded open set containing the unit ball $B_{1}$. If

$$
\gamma^{s}(x)=\left\{\begin{array}{cl}
1 & \text { if }|x| \leq 1 \\
\frac{1}{s}|x|+\frac{s-1}{s} & \text { if }|x|>1
\end{array}\right.
$$

then $\Omega^{s}=B_{1}$ for every $s \in \mathbb{N}$, while $\Omega^{\infty}=\Omega$.

Proof. 1) Define

$$
\chi^{s}(x)= \begin{cases}0 & \text { if } x \in \Omega^{s} \\ 1 & \text { if } x \notin \Omega^{s}\end{cases}
$$


and similarly for $\chi^{\infty}$. Note that because of the convergence of $\gamma^{s}$ to $\gamma^{\infty}$, we have that, at almost all points where $\chi^{\infty}(x)=1$ (i.e. $\gamma^{\infty}(x)>1$ ) and for large enough $s, \chi^{s}(x)=1$ and thus

$$
\lim _{s \rightarrow \infty} \chi^{s}(x)=\chi^{\infty}(x) \text {, a.e. } x \notin \Omega^{\infty} .
$$

Moreover, trivially, $\liminf _{s \rightarrow \infty} \chi^{s}(x) \geq \chi^{\infty}(x)=0$, a.e. $x \in \Omega^{\infty}$ and therefore

$$
\liminf _{s \rightarrow \infty} \chi^{s}(x) \geq \chi^{\infty}(x) \text {, a.e. } x \in \Omega .
$$

Therefore by Fatou's lemma

$$
\begin{aligned}
\liminf _{s \rightarrow \infty}\left[\operatorname{meas}(\Omega)-\operatorname{meas}\left(\Omega^{s}\right)\right] & =\liminf _{s \rightarrow \infty} \int_{\Omega} \chi^{s}(x) d x \\
& \geq \int_{\Omega} \chi^{\infty}(x) d x=\operatorname{meas}(\Omega)-\operatorname{meas}\left(\Omega^{\infty}\right)
\end{aligned}
$$

which gives the upper semicontinuity.

2) Let $B_{\varepsilon}=\left\{x \in \mathbb{R}^{n}:|x| \leq \varepsilon\right\}$ and for $A \subset \mathbb{R}^{n}$ define

$$
A+B_{\varepsilon}=\left\{x \in \mathbb{R}^{n}: x=y+z \text { with } y \in A \text { and }|z| \leq \varepsilon\right\} .
$$

The Hausdorff distance between two sets is then defined as

$$
d(A, B)=\inf \left\{\varepsilon \geq 0: A \subset B+B_{\varepsilon}, B \subset A+B_{\varepsilon}\right\} .
$$

Observe (see below) that since $\gamma^{s}$ and $\gamma^{\infty}$ are gauges then

$$
d\left(\Omega^{s}, \Omega^{\infty}\right) \rightarrow 0, \text { as } s \rightarrow \infty,
$$

and therefore (see Theorem 6.2.17 in [13])

$$
\text { meas }\left(\Omega^{\infty}\right)=\lim _{s \rightarrow \infty} \text { meas }\left(\Omega^{s}\right) \text {. }
$$

We now establish (16). We will prove that for every $\varepsilon>0$ we can find $s$ sufficiently large so that

$$
\Omega^{\infty} \subset \Omega^{s}+B_{\varepsilon}, \Omega^{s} \subset \Omega^{\infty}+B_{\varepsilon} .
$$

Assume without loss of generality that $x_{0}=0$. Since $\Omega$ is bounded and $\gamma^{s}$ are gauges that converge almost everywhere to a gauge $\gamma^{\infty}$, the convergence is, in fact, uniform. Furthermore there exist $m, M>0$ so that

$$
m|x| \leq \gamma^{s}(x), \gamma^{\infty}(x) \leq M|x|, \forall x \in \Omega,
$$

and, for $s$ sufficiently large,

$$
\left|\gamma^{s}(x)-\gamma^{\infty}(x)\right| \leq \varepsilon^{2}, \forall x \in \Omega .
$$

Let $x \in \Omega^{\infty}$, i.e. $\gamma^{\infty}(x) \leq 1$, and choose $\delta>0$ such that

$$
\frac{\varepsilon^{2}}{1+\varepsilon^{2}} \leq \delta \leq m \varepsilon
$$

and observe that

$$
\begin{aligned}
\gamma^{s}((1-\delta) x)=(1-\delta) \gamma^{s}(x) & \leq(1-\delta)\left(\gamma^{\infty}(x)+\varepsilon^{2}\right) \leq(1-\delta)\left(1+\varepsilon^{2}\right) \leq 1, \\
|\delta x| & \leq \delta \frac{\gamma^{\infty}(x)}{m} \leq \frac{\delta}{m} \leq \varepsilon
\end{aligned}
$$

Therefore $x=(1-\delta) x+\delta x \in \Omega^{s}+B_{\varepsilon}$ which is the first inclusion in (17). The second one being proved in a similar manner, we have the claim. 


\section{ACKNOWLEDGMENTS}

The research of Bernard Dacorogna was partially supported by the Fonds National Suisse (21-61390-00). The research of Paolo Marcellini was partially supported by the Italian Ministero dell'Istruzione, dell'Università e della Ricerca (MIUR) and through the contribution of the Italian Ministero degli Affari Esteri, Direzione Generale per la Promozione e la Cooperazione Culturale (MAE).

\section{REFERENCES}

[1] M. Bardi and I. Capuzzo Dolcetta, Optimal control and viscosity solutions of HamiltonJacobi-Bellman equations, Birkhäuser, 1997. MR 99e:49001

[2] F. Camilli and A. Siconolfi, Maximal subsolutions for a class of degenerate Hamilton-Jacobi problems, Indiana Univ. Math. J., 48 (1999), 1111-1131. MR 2001a:49028

[3] P. Cardaliaguet, B. Dacorogna, W. Gangbo and N. Georgy, Geometric restrictions for the existence of viscosity solutions, Annales Institut Henri Poincaré, Analyse Non Linéaire, 16 (1999), 189-220. MR 99k:35023

[4] B. Dacorogna, R. Glowinski and T.W. Pan, Numerical methods for the solution of a system of eikonal equations with Dirichlet boundary conditions, Comptes Rendus Acad. Sci. Paris, 336 (2003), 511-518.

[5] B. Dacorogna and P. Marcellini, General existence theorems for Hamilton-Jacobi equations in the scalar and vectorial case, Acta Mathematica, 178 (1997), 1-37. MR 98d:35029

[6] B. Dacorogna and P. Marcellini, Implicit partial differential equations, Progress in Nonlinear Differential Equations and their Applications, 37, Birkhäuser, Boston, 1999. MR 2000f:35005

[7] L. Hörmander, Notions of convexity, Birkhaüser, 1994. MR 95k:00002

[8] H. Ischii and P. Loreti, Relaxation in an $L^{\infty}$-optimization problem, Proc. Royal Soc. Edinburgh, 133 (2003), 599-615.

[9] H. Ischii and M. Ramaswamy, Uniqueness results for a class of Hamilton-Jacobi equations with singular coefficients, Comm. Par. Diff. Eq., 20 (1995), 2187-2213. MR 96k:35026

[10] P. L. Lions, Generalized solutions of Hamilton-Jacobi equations, Research Notes in Math. 69, Pitman, London, 1982. MR 84a:49038

[11] P. Marcellini, Non convex integrals of the calculus of variations, in: Methods of nonconvex analysis, ed. Cellina A., Lecture Notes in Math. 1446, Springer-Verlag, Berlin, 1990, 16-57. MR 91j:49002

[12] R.T. Rockafellar, Convex analysis, Princeton University Press, Princeton, 1970. MR 43:445

[13] R. Webster, Convexity, Oxford University Press, Oxford, 1994. MR 98h:52001

Départment de Mathématiques, École Polytechnique Fédérale de Lausanne, 1015 Lausanne, Switzerland

E-mail address: bernard.dacorogna@epfl.ch

Dipartimento di Matematica U. Dini, Università di Firenze, Firenze, Italy

E-mail address: marcell@math.unifi.it 